\title{
A checklist of Polygala (Polygalaceae) in the Flora of Southern Africa region with notes on types
}

\author{
ESTRELA FIGUEIREDO ${ }^{1,2}$, GIDEON F. SMITH $^{2,3,4} \&$ JORGE PAIVA $^{2}$
}

${ }^{1}$ Department of Botany, P.O.Box 77000, Nelson Mandela Metropolitan University, Port Elizabeth, 6031

South Africa.Email: estrelafigueiredo@hotmail.com (corresponding author)

${ }^{2}$ Centre for Functional Ecology, Departamento de Ciências da Vida, Universidade de Coimbra, 3001-455

Coimbra, Portugal.

${ }^{3}$ Office of the Chief Director: Biosystematics Research \& Biodiversity Collections, South African National Biodiversity Institute, Private Bag X101, Pretoria, 0001 South Africa.

${ }^{4}$ H.G.W.J. Schweickerdt Herbarium, Department of Plant Science, University of Pretoria, Pretoria, 0002 South Africa.

\begin{abstract}
As part of a revision of Polygala in the Flora of Southern Africa region, the types of all the names relevant for the region were assessed. A catalogue of the names and their types is provided along with information on the availability of online images of these types. For 22 accepted taxa, no images of the types of the names are available online. Twenty lectotypes are designated here.
\end{abstract}

\section{INTRODUCTION}

As a first step towards a revision of the genus Polygala Linnaeus (1753: 701) (Polygalaceae) in the Flora of Southern Africa region (Botswana, Lesotho, Namibia, South Africa, and Swaziland), the typification of names published in the genus for this region was compiled and analysed. As a result, 20 lectotypes are designated here. We provide a list of the names and their types, along with information on the images of the types that are available online. For 22 accepted taxa, no images of the types of the names could be located on the web.

\section{MATERIAL AND METHODS}

With some corrections, the taxonomy of Polygala followed here coincides with Paiva (1998). The specimens cited are those seen by one of the authors (Paiva 1998) during the course of his revision of the genus. Specimens marked '!' were examined in the scope of this paper. The following online resources were consulted: JSTOR Global Plants (2013), as well as the Zürich Herbaria Database 
(2013), Geneva Herbaria Catalogue (2013), Kew Herbarium Catalogue (2013), The Linnean Collections (2013) and Uppsala Herbarium (UPS) Database (2013). The acronyms of herbaria follow Thiers (2013).

\section{RESULTS}

The information on the types of 209 names was reviewed. Corrections were made to type citations in earlier literature where lectotypes were often confusingly referred to as holotypes. In Art. 9.9 of the International Code of Nomenclature for algae, fungi and plants (ICN) it is stated that the use of a term in a sense other than in which it is defined in the ICN is treated as an error to be corrected (McNeill et al. 2012). In some cases, it was found that previously designated lectotypes included more than one specimen. In such cases, a second-step lectotype is designated here to narrow down the lectotype to a single specimen, according to Art. 9.17 of the ICN (McNeill et al. 2012).

R. Chodat (1865-1934), based at the University of Geneva, was the most prolific author of southern African Polygala names, with 52 names published. His herbarium was destroyed by a fire in 1898 (Anonymous 1899) which may explain why many duplicates of the types of his names could not be located.

W.H. Harvey was based at TCD when he produced the account of Polygala for Flora Capensis (Harvey \& Sonder 1860). He referred to material under collector and number without mention of the herbarium where it was held. He did however cite herbaria holding duplicates as TCD, K ("Hooker"), and HBG ("Sonder"). In addition to these herbaria, several others hold duplicates of these collections. Therefore automatically deducing that the holotypes of the names published by Harvey are at TCD is not the correct approach, as it is clear that Harvey examined several duplicates and did not select one as type. The existence of numerous syntypes and isosyntypes further complicates the typification of Harvey's names (see Smith \& Figueiredo 2013). Twenty lectotypes are designated here and provided in the list below.

To facilitate future use of this work, the online availability of images of the types is recorded. Twenty-two accepted taxa do not have any image of the types of the names accessible in the consulted online catalogues.

\section{LIST OF NAMES AND TYPES}

Names are listed alphabetically. Synonyms are in italics and accepted names in bold italics. For specimens accessible online, their reference (mostly a barcode) is given instead of the herbarium acronym. Given that the herbarium acronym does not match the acronym included in the barcode in all cases, sometimes both are cited. 
Polygala abbreviata Markötter (1930: 28) [=P. transvaalensis]

Type:-SOUTH AFRICA. Free State/KwaZulu-Natal: Oliviershoek Pass, Thode 4624 (holotype NBG0199218-0!).

Polygala affinis Candolle (1824: 322)

Type:-SOUTH AFRICA. Without locality, Anonymous s.n. (holotype G).

Note:-Paiva (1998: 268) indicated a specimen at G as type of $P$. affinis. In the protologue of $P$.

affinis Candolle refers to a specimen at ' $h$. Deless.', now part of the G herbarium, where the type of this name must be located.

Polygala africana Chodat (1893: 168)

Type:-ANGOLA. Welwitsch 1009 [1109] (holotype B†, lectotype LISU [designated by Paiva 1998: 161], isolectotype BM)

Polygala agnipila Gandoger (1913: 455) [=P. affinis $]$

Type:-SOUTH AFRICA. Western Cape: Saron, Schlechter 4879 (holotype LY?, isotype PRE!).

Polygala albida Schinz (1888: 53)

Type:- NAMIBIA. Olukonda, Schinz 506 (lectotype K000231662 [designated by Paiva 1998: 190]).

Note:-Schinz did not cite the collection number in the protologue of Polygala albida, he only cited the collecting locality, but later Chodat (1893: 339) cited the specimen Schinz 506 when he described $P$. livingstoniana (a synonym of this species). Chodat was not aware that the name $P$. albida had already been published based on that same collection, as he did not mention that species name. Paiva (1998) stated that the holotype of P. albida is Schinz $506(\mathrm{G})$ and that it no longer exists. However, Chodat referred to 'Herb. Schinz', which is deposited at Z, therefore citations of $\mathrm{G}$ could be incorrect. No collections of Polygala by Schinz could be found in either the Geneva Herbaria Catalogue (2013) or in the Zürich Herbaria Database (2013).

Polygala amatymbica Ecklon \& Zeyher (1835: 24)

Type:-SOUTH AFRICA. Eastern Cape: Tambukiland, Klipplaatrivier, Ecklon \& Zeyher 182 (lectotype K [designated by Paiva 1998: 242], isolectotypes CGE, E, JE00003229!, M0104330!, OXF, S08-15988!,S08-15989!, SAM0048687-0!, TCD0001528!, W). 
Polygala amoena Thunberg (1800: 120) [=P. myrtifolia $]$

Type:-SOUTH AFRICA. Thunberg, s.n. (lectotype UPS Nr 16205! designated here, isolectotype UPS Nr 16204!).

Polygala arcuata Chodat (1912: 333) non Hayata (1908), nom. illeg. [=P. rodrigueana] (see Paiva 1998)

Type:-Same as $\boldsymbol{P}$. rodrigueana.

Polygala armata Chodat (1912: 327) [=P.leptophylla var. armata $]$

Type:--Same as P. leptophylla var. armata.

Polygala asbestina Burchell (1822: 543)

Type:-SOUTH AFRICA. Eastern Cape: Gariep, Burchell 2030 (holotype K000231659! isotypes K000231658!, PRE0255281-0).

Polygala asperifolia Chodat (1912: 323) [=P. stenopetala $]$

Type:-SOUTH AFRICA. Mpumalanga: Barberton, Galpin 844 p.p. (holotype Z).

Note:-The holotype of $P$. asperifolia has not been located at Z.

Polygala attenuata Loddiges (1824: t. 1000) non Nuttall (1818: 90) nom. illeg. [=P. fruticosa $]$ Type:- Illustration in Loddiges (1824: t.1000) lectotype designated here.

Polygala bicornis Burchell ex Chodat (1896: 236) [=P. schinziana $]$

Type:-BOTSWANA? Bechuanaland, Chovi [Chobe] Desert, Burchell 2351 (holotype K000231793!).

Note:-The type of P. bicornis has been recorded as originating in South Africa, but considering the locality given, it could be a collection from Botswana.

Polygala borboniifolia Burchell ex Candolle (1824: 322, as'borboniaefolia’) [=P. fruticosa $]$ Type:-SOUTH AFRICA. Burchell 6861 (lectotype G-DC G00210312! [designated by Paiva 1998: 281 as 'holo- '], isolectotypes K000231643!, NY00444473!). 
Polygala bowkerae Harvey in Harvey \& Sonder (1860: 92)

Type:-SOUTH AFRICA. Eastern Cape: Graaff-Reinet, Bowker s.n. (holotype K000231655!).

Note:-Bowker s.n. (TCD0001526!), a fragment, which possibly is a duplicate of the holotype.

Polygala brachyphylla Chodat (1893: 417)

Type:-SOUTH AFRICA. Western Cape: Clanwilliam, Blaawberg, Drège 7202 (lectotype

K000231654! [designated by Paiva 1998: 268 as 'holo-'], isolectotypes BM, PRE0261548-0!, S08$16336 !)$.

Polygala bracteolata Linnaeus (1753: 702)

Type:-SOUTH AFRICA. Coll. details unknown (lectotype SBT L98, image! [designated by

Figueiredo et al. 2013] ).

Polygala bracteolata var. racemosa Harvey in Harvey \& Sonder (1860: 84) [=P. bracteolata] Syntypes:-SOUTH AFRICA. Ecklon \& Zeyher 158 (S08-16474!, S08-16471!, W), 159 (OXF, S08-16476!, S08-16477!, W), 160 (E, S08-16469!, S08-16466!, W).

Note:- Only these three collection numbers are cited in the protologue of this variety. The Herbaria cited by Harvey are Thunberg's, TCD, K, Sonder and 'others'.

Polygala bracteolata var. umbellata Harvey in Harvey \& Sonder (1860: 84) [=P. bracteolata] Type:-SOUTH AFRICA. Ecklon \& Zeyher 156 (M, S08-16465!).

Note:- Only this collection is cited in the protologue of this variety. The Herbaria cited by Harvey are Thunberg's, TCD, K, Sonder and 'others'. A duplicate, S S08-16462, recorded in JSTOR as Ecklon \& Zeyher 156 appears to be a Drège collection.

Polygala brevifolia Harvey in Harvey \& Sonder (1860: 90) non Nuttall (1818: 89) nom. illeg. [=P. brachyphylla]

Type:-Same as $\boldsymbol{P}$. brachyphylla.

Polygala burmannii Candolle (1824: 322) [=P. affinis $]$

Type:-SOUTH AFRICA. Burchell 6437 (lectotype K000231663! [designated by Paiva 1998: 268 as 'holo-'], G-DC, PRE0259681-0!). 


\section{Polygala calycina Presl (1845: 445) [=P. bracteolata $]$}

Type:-SOUTH AFRICA. Drège s.n. (holotype not determined)

Note:-There are many collections Drège s.n. and it could not be determined which of them were examined by Presl. Paiva (1998) cited collections from Paarlberg (BM, K) and from Caledon (E, W). Presl types are mostly at PR (Prague).

Polygala capillaris Meyer ex Harvey in Harvey \& Sonder (1860: 93)

Type:-SOUTH AFRICA. Eastern Cape: between the Omsamculo [Umzimkulu] and Omcomas [Umkomaas], Drège s.n. (lectotype K000231653! [designated by Paiva 1998: 158 as 'holo-']). Notes:- Several specimens of P. capillaris labelled 'Drège s.n.' are often listed as types (e.g., BM, CGE, E, LD, NY00444474, P, PRE0264215-0, REG000530, TCD0001529, TUB001884, W), but as a result of lacking collecting numbers they cannot be considered as duplicates of the holotype. However, TCD is the first Herbarium cited in the protologue and it was the Herbarium where Harvey was based. That specimen is therefore likely to be part of the original material. A specimen Drège s.n. at PRE (PRE0264215-0) has a handwritten indication of type and was received from K. The specimen Drège s.n. at K (K000231653) has a handwritten note stating that a duplicate was removed and sent to PRE.

On the same sheet, PRE0264215-0, there is another specimen, from the Magaliesberg, South Africa, which appears to be a Burke \& Zeyher collection that Harvey cited in the protologue of this species. That collection was later considered to be of another species, Polygala africana, and was excluded from $P$. capillaris by Exell (1960).

Polygala capillaris var. angolensis Oliver (1868: 131) [=P. spicata $]$ Type:-ANGOLA. Welwitsch 1027 (holotype LISU, isotypes BM, COI).

Polygala carmichaelii Harvey in Harvey \& Sonder (1860: 19) [=P. lehmanniana $]$

Type:-SOUTH AFRICA. Western Cape: Cape of Good Hope, Carmichael s.n. (lectotype K000231825! [designated by Paiva 1998: 267 as 'holo-']).

Note:-Under Polygala carmichaelii, Harvey \& Sonder (1860) cited the collection Carmichael s.n., with duplicates in three herbaria ('TCD, Hook., Sond.').

Polygala cernua Thunberg (1800: 120) [=P. virgata $]$

Type:-SOUTH AFRICA. Thunberg s.n. (holotype UPS Nr 16209!). 
Polygala chloroptera Chodat (1893: 375) [=P. serpentaria $]$

Type:-SOUTH AFRICA. KwaZulu-Natal: Natal, Rehmann s.n. (holotype Z).

Note:- Searches for the holotype at $\mathrm{Z}$ were fruitless.

Polygala ciliatifolia Turczaninow (1854: 347) [=P. umbellata $]$

Type:-SOUTH AFRICA. Ecklon \& Zeyher 106 (holotype KW001000268! isotypes M, W).

Note:-Although Paiva designated the M specimen as lectotype (Paiva 1998: 271), it is considered that all types of Turczaninow are at Kiew (KW), Ukraine.

Polygala ciliatifolia var. mundtiana Chodat (1893: 428) [=P. umbellata $]$

Type:-SOUTH AFRICA. Ecklon \& Zeyher 167 (lectotype M M0153118! designated here,W).

Polygala cluytioides Burchell ex Candolle (1824: 322) [=P.myrtifolia $]$

Type:-SOUTH AFRICA. Somerset, Burchell 3326 (lectotype G-DC G00210311! [designated by

Paiva 1998: 279 as 'holo- '], K000231813).

Polygala confusa MacOwan (1890: 385) non Hasskarl (1864: 165) nom. illeg. [ $=\boldsymbol{P}$.

macowaniana]

Type:-SOUTH AFRICA. Eastern Cape: Griqualand East, Malowe Mts, Tyson 2082 (lectotype

K000231818! [designated by Paiva 1998: 185 as 'holo-'], E, PRE!, SAM0029323-2!,

SAM0029323-1!). Syntypes: Herb. Norm. Austro-Afr. 890 (BM, BOL136751!, E, K000231819!,

W); MacOwan 1266, 1325; Baur 17 (K, SAM0029321-0!); Cooper 165 (BM, K, W), 301 (BM,

E, PRE!, W), 1914, 1926 (K); Natal [now KwaZulu-Natal], Gerrard 1202 (BM, K, W); Wood

1805 (not located).

Polygala confusa var. acutifolia Chodat (1893: 396), nom. illeg. [=P. macowaniana $]$

Syntypes:-SOUTH AFRICA. KwaZulu-Natal: Baur 17 (K, SAM0029321!-0),, Gerrard 1202

(BM, K, W).

Polygala confusa var. canescens Chodat (1893: 397), nom. illeg. [=P. macowaniana]

Type:-SOUTH AFRICA. MacOwan $666 b(\mathrm{BM})$.

Polygala confusa forma villosiuscula Hayek (1900: 42) nom. illeg. [=P. macowaniana]

Type:-SOUTH AFRICA. Eastern Cape: Komga, Krook 1476 (holotype W18970008931!). 
Polygala cordifolia Thunberg (1800: 120) [=P.fruticosa $]$

Type:-SOUTH AFRICA, s.c. s.n. (lectotype UPS Nr 16213 designated here, isolectotype UPS $\mathrm{Nr} 16214)$.

Polygala dasyphylla Levyns (1955: 39)

Type:-SOUTH AFRICA. Western Cape: Bredasdorp, Levyns 9763 (holotype BOL136746!-, isotype BOL136747!).

Polygala declinata (Harv.) Meyer ex Paiva (1998: 210). Basionym: Polygala hispida var. declinata Harvey in Harvey \& Sonder (1860: 91)

Type:-SOUTH AFRICA. Cape, between Omsamcaba und Omtendo, Drège s.n. (lectotype BM [designated by Paiva 1998: 210]).

Note:- Several unnumbered specimens collected by Drège are possible duplicates of the lectotype (E, TCD, K, HBG, S S08-16451, W).

Polygala decora Sonder (1850: 14) [=P. virgata var. decora]

Type:- Same as $\boldsymbol{P}$. virgata var. decora.

Polygala dietrichiana D.Dietrich (1847: 905)

Type:-Unknown.

Note:-A replacement name for P. venulosa A. Dietrich (1834) non Sibthorp \& Smith (1813), a nom. illeg. The name was based on a cultivated plant (see Paiva 1998). If the type exists, it could be at HBG where A. Dietrich's types are held.

Polygala diffusa A.Dietrich (1834: 114) [=P.fruticosa $]$

Type:-Unknown.

Note:-Name based on a cultivated plant (see Paiva 1998).

Polygala dodii Schlechter (1898: 24) [=P. garcinii $]$

Type:-SOUTH AFRICA. Wolley Dod 813 (holotype K).

Paiva (1998) indicated that the holotype is at K. Schlechter' herbarium was mostly destroyed in Berlin. It is not known if it included a duplicate of Wolley Dod 813. 
Polygala dregeana Presl (1845: 444) [=P.fruticosa $]$

Type:-SOUTH AFRICA. Drège s.n. (holotype not located)

Note:-There are many Drège s.n. collections and it could not be determined which of them were examined by Presl. Presl types are mostly at PR (Prague).

Polygala durbanensis Chodat (1893: 401) [?=P. hottentotta]

Type:-SOUTH AFRICA. KwaZulu-Natal: Natal, Rehmann 8766 (holotype Z?).

Note:-Rehmann 8766 is not listed in the Zürich Herbaria Database (2013).

Polygala eckloniana Presl (1845: 445) non Lehmann (1830: 8), nom. illeg. [=P. teretifolia]

Type:-SOUTH AFRICA. Ecklon \& Zeyher 154 (BREM, K, LD, M0153112!, OXF, PR?, PRE!, S08-16662!, S08-16663!, W).

Polygala eckloniana Lehmann (1830: 8) [=P. ericaefolia $]$

Type:-SOUTH AFRICA. Ecklon s.n. (holotype HBG).

Polygala ephedroides Burchell (1822: 465)

Type:-SOUTH AFRICA. Eastern Cape: Griqualand, Lower Campbell, Burchell 1793 (holotype K000231651!-, isotypes K000231649!, M0152982!).

Polygala ericaefolia Candolle (1824: 323)

Type:-SOUTH AFRICA. Western Cape: Goukamma Station, Mossel Bay, Burchell 5614 (lectotype G-DC G00210254! [designated by Paiva 1998: 270 as 'holo-'], isolectotypes BOL136743!, K000231645!, K000231647!, M, PRE0255282-0!, W).

Polygala ericaefolia var. eckloniana (Lehm.) Harvey in Harvey \& Sonder (1860: 87) [=P. ericaefolia]. Basionym: Polygala eckloniana Lehmann (1830: 8)

Type:- Same as P. eckloniana Lehm.

Polygala erioptera De Candolle (1824: 326)

Type:-SENEGAL. Bacle s.n. (lectotype G-DC G00135151! [designated by Paiva 1998: 172]).

Polygala erubescens Meyer ex Chodat (1893: 398)

Type:-SOUTH AFRICA. Drège s.n. (holotype P). 
Note:- Several unnumbered specimens of Polygala erubescens collected by Drège (kept at BM, CGE00089!, E, HBG508684!, K000231842!, REG000529!, S08-16431!, S08-16432! \& W) are possible duplicates of the holotype.

Polygala esterae Chodat (1912: 331) [=P. gazensis $]$

Type:-SOUTH AFRICA. Bachmann 749 (lectotype B [designated by Paiva 1998: 275]).

Note:- In the protologue of Polygala esterae Chodat cites the collection Bachmann 745 as type.

Paiva (1998: 275) designated the specimen with the number 749 at B as lectotype. However, elsewhere in the same reference Paiva (1998: 330) notes that the correct number is 739 . These specimens could not be examined online.

Polygala fallax Hayek (1900: 43) non Chodat (1889: 104), nom. illeg. [=P. houtboshiana] Type:-SOUTH AFRICA. Krook (Plantae austro-africanae, Penther) 1477 (holotype W W18970008936!).

Polygala fruticosa Bergius (1767: 183)

Type:-SOUTH AFRICA. Grubb s.n. (holotype SBT10181!).

Polygala garcinii Candolle (1824: 323)

Type:-SOUTH AFRICA. Western Cape: between Cape Town and Table Mountain, Burchell 20 (‘2870’) (lectotype G-DC G00210286 [designated by Paiva 1998: 270], isolectotype K).

Polygala gazensis Baker in Rendle et al. (1911:24)

Type:-ZIMBABWE. Swynnerton 632 (holotype BM000564822!-, K000231675!).

Polygala genistoides Poiret in Lamarck (1804: 492) [=P. virgata]

Type:-SOUTH AFRICA. S.c. s.n. (holotype P-LA).

Polygala genistoides var. spartioides Burchell ex Candolle (1824: 323) [=P. virgata $]$

Type:-SOUTH AFRICA. Burchell 4499 (lectotype K K000231772! designated here, isolectotype G-DC G00210260)

Note:-The collection number is wrongly cited in the protologue as 4449 . 
Polygala genistopsis Chodat (1893: 405) [=P. virgata $]$

Type:-SOUTH AFRICA. Krebs s.n. (holotype B $\dagger$ ).

Polygala gerrardii Chodat (1896: 237)

Type:-SOUTH AFRICA. KwaZulu-Natal: Zululand, Gerrard 1201 [1281] (lectotype

K000231634! [designated by Paiva 1998: 241], BM000564940!, NH0005713-0!, W). Syntypes:

Natal, Inanda, Wood 1394 (BM, K000231635!), 3481 (K000231636!).

Polygala gracilenta Burtt Davy (1926: 48, 134).

Type:-SOUTH AFRICA. Eastern Cape: Winterberg, Ecklon \& Zeyher 163 (lectotype

K000231631! [designated by Paiva 1998: 217], isolectotypes HBG508699!, M, PRE0255285-0!,

S08-16604!, S08-16606!, W).

Polygala gracilipes Harvey in Harvey \& Sonder (1860: 90) pro parte (see Paiva 1998: 269)

Type:-SOUTH AFRICA. Drège 7191 (lectotype K [designated by Paiva 1998: 269 as 'holo-'], isolectotypes BM000564935!, COI, E, MO-251880!, P, PRE0023528-0!, S08-16445!, W).

Syntypes: SOUTH AFRICA, Drège 7192 (TCD0001525!, MO-251879!, S08-16446!).

Polygala gracilipes var. angustifolia Chodat (1893: 410) [=P. bowkerae]

Type:-SOUTH AFRICA. Drège 7192 (holotype K -, isotypes COI, P, W).

Polygala gracilipes var. macrostachya Chodat (1893: 410) [=P. bowkerae]

Type:-SOUTH AFRICA. MacOwan 997 (holotype K-, isotypes COI, E).

Polygala grandiflora Loddiges (1827: t.1227) non Walter (1788: 179) nom. illeg. [=P. myrtifolia ] Type:-Illustration in Loddiges (1827: t.1227), lectotype designated here.

Note:-Paiva (1998) suggested a potential neotype, but that is not an option as original material (an illustration) exists.

Polygala guerichiana Engler (1894: 141) 'gürichiana'

Type:-NAMIBIA. Gürich 64 (holotype B†). 
Polygala gymnoclada MacOwan (1890: 385)

Type:- SOUTH AFRICA. Eastern Cape: Griqualand East, Kokstad Dist., Tyson 1120 (lectotype K000231848! [designated by Paiva 1998: 256], isolectotypes BOL136750!, SAM0000975-0!, SAM0005574-2!, SAM0005574-1!). Syntypes: Baur 63 (K000231846!), 243 (K000231845!),

Cooper 927 (K000231847!, CGE00090!), Herb. Norm. Austr.Afr. 884 (K000231848!, SAM0029325A-0!).

Polygala hamata Burtt Davy (1926: 132) [=P. uncinata $]$

Type:- Same as $\boldsymbol{P}$. uncinata.

Note:- See under $\boldsymbol{P}$. uncinata.

Polygala harveyana Chodat (1893: 412) [=P. refracta $]$

Type:-SOUTH AFRICA. Burchell 6058 pp (holotype K000231797!).

Note:-Burchell 6058 is a mixed collection (Levyns 1955). On the K sheet there are also specimens of P. garcinii (K000231796).

Polygala hispida Burchell ex Candolle (1824: 323)

Type:-SOUTH AFRICA. Eastern Cape: Port Elizabeth, Burchell 4473 (lectotype K000231844!

[designated by Paiva 1998: 210 as 'holo-'], isolectotypes G-DC G00210448!, P00375264!, PRE!).

Polygala hispida var. declinata Harvey in Harvey \& Sonder (1860: 91) [=P. declinata]

Type:- Same as $\boldsymbol{P}$. declinata.

Note:-See under $\boldsymbol{P}$. declinata.

Polygala hispidula Pres1 (1845: 445) [=P. peduncularis]

Type:-SOUTH AFRICA. Drège 7188 (lectotype? BM000564937!, isolectotype? PRE0256350$0 !)$.

Note:-The specimen BM000564937 is listed as lectotype in JSTOR Global Plants (2013).

However, we could not locate where in the literature it was accordingly designated. It is not known if a holotype exists at PR.

Polygala hottentotta Presl (1845: 445)

Type:-SOUTH AFRICA. Drège 7194 (lectotype W [designated by Paiva 1998: 259], isolectotype K000231838!). 
Note:-We could not determine if there is a holotype at PR, which would take precedence over the lectotype.

Polygala houtboshiana Chodat (1893: 400)

Type:-SOUTH AFRICA. Limpopo: Transvaal, Houtbosh, Rehmann 6347 (holotype P, isotype K000231837!).

Note:-Paiva (1998) cites the holotype as being at P.

Polygala humilis Loddiges (1820: tab. 420)

Type:-Illustration in Loddiges (1820: tab. 420), lectotype designated here.

Polygala imbricata Hayek (1900: 45) [=P.fruticosa $]$

Type:-SOUTH AFRICA. Penther 1519 (M0153000!, W).

Note:-The holotype is probably the specimen at W, where Hayek was based.

Polygala illepida Meyer ex Harvey in Harvey \& Sonder (1860: 92)

Type:-SOUTH AFRICA. Eastern Cape: Uitenhage, Ecklon \& Zeyher 178 (lectotype K000231833! [designated by Paiva 1998: 245], isolectotypes LD, S08-16489!, S08-16487!, SAM0014193-0!, TCD, W). Syntype: Drège s.n. (not located).

Note:-Several specimens are possible duplicates of the syntype Drège s.n.: HBG508682!, K000231835!, K000231836!, TUB001900!, S08-16483! \& S08-16485!.

Polygala intermedia Candolle (1824: 322) [=P. peduncularis $]$

Type:-SOUTH AFRICA. S.c. 14 (holotype G-DC).

Polygala kalaxariensis Schinz (1888: 52)

Type:-NAMIBIA. Lewisfonteyn, Kalaxari, Schinz 505 (holotype Z†, lectotype K000231832! [designated by Paiva 1998: 231], isolectotype GRA0001789-1!).

Note:-The holotype of $P$. kalaxariensis has not been located at $\mathrm{Z}$ where it was searched for. It was considered destroyed by Paiva (1998), who designated the specimen at K as lectotype. 
Polygala krumanina Burchell ex Ficalho \& Hiern (1881: 16)

Type:-SOUTH AFRICA. Northern Cape: Kruman [Kuruman], Burchell 2425 (lectotype

K000231829! [designated by Paiva 1998: 242]). Syntypes: South Africa, Burchell 2474

(K000231831!) \& 2599 (K000231830!); ?Angola, Serpa Pinto 29 (LISU).

Polygala lanata Meyer ex Meisner (1842: 469) [=P. hispida $]$

Type:-SOUTH AFRICA. Cape, Drège s.n. (lectotype K K000231841! designated here).

Note:-Several unnumbered specimens of Polygala hispida collected by Drège (kept at BM,

BREM, CGE, E, HBG508685!, REG000526!, TUB001899!, W) are possible duplicates of the lectotype.

Polygala langebergensis Levyns (1955: 39)

Type:-SOUTH AFRICA. Western Cape, Riversdale, Garcia's Pass, Levyns 8988 (holotype

BOL136745!, isotype BOL136744!, PRE0810044-0!).

Polygala lasiopoda Presl (1845: 445)

Type:-SOUTH AFRICA. Drège s.n. (holotype not located)

Note:-The type could not be identified among the several unnumbered collections of Drège. Presl types are mostly at PR (Prague).

Polygala lasiosepala Levyns (1955: 21)

Type:-SOUTH AFRICA. Western Cape: Clanwilliam, Nardouw Mt, Stokoe 8450 (holotype BOL).

Polygala latifolia Ker Gawler (1822: pl. 645) [=P.fruticosa $]$

Type:-Illustration in Ker Gawler (1822: tab. 645), holotype.

Polygala laxa Thunberg (1800: 121) [?=P. ludwigiana $]$

Type:-SOUTH AFRICA. Thunberg s.n. (holotype UPS Nr 16222, isotype LD1238064!).

Note:-An isotype of P. laxa at LD is a specimen of P. ludwigiana, as confirmed on labels added by both Levyns and Paiva. If the holotype at UPS proves to be the same as the isotype, then the name $P$. laxa has priority over $P$. ludwigiana. The holotype is not available for examination online, nor on loan. It is available only on microfiche ( $\mathrm{Nr} 16222)$, but the quality of this medium is insufficient for the specimen to be assessed. 
Polygala leendertziae Burtt Davy (1926: 48, 133, 135)

Type:-SOUTH AFRICA. Gauteng: Witwatersrand, Leendertz 1732 (holotype K000231828!, isotype PRE0712072-0!).

Polygala lehmanniana Ecklon \& Zeyher (1835: 23)

Type:-SOUTH AFRICA. Western Cape: Worcester, Tulbagh Kloof, Ecklon \& Zeyher 177 (lectotype K000231827! [designated by Paiva 1998: 267 as 'holo-'], isolectotypes M0104324!, MO-251878!, OXF, S08-16502!, SAM0029300-0!).

Polygala lehmanniana var. pteropus Harvey in Harvey \& Sonder (1860: 20) [=P. lehmanniana] Type:-SOUTH AFRICA. Western Cape: Cape of Good Hope, Carmichael in Herb. Brown s.n. (lectotype K K000231826! [designated by Paiva 1998: 267 as 'holo-']).

Note:-Under Polygala lehmanniana var. pteropus Harvey \& Sonder (1860) cite a collection Carmichael s.n., with duplicates in three herbaria ('TCD, Hook., Sond.'). Paiva (1998) considered that to be a possible mistake and referred to the type as the collection Brown s.n. (K), however the collection shows a label indicating 'Herb. Brown' and a determination label as var. pteropus, which indicates that there was no mistake in the protologue, the type having been collected by Carmichael and deposited in Brown's herbarium. Carmichael s.n. at TCD (TCD0002084) and S (S08-16504) are possible duplicates.

Polygala leptophylla Burchell (1822: 400) Type:-SOUTH AFRICA. Eastern Cape: Griqualand: Vaal, Blaauwbosh Drift, Burchell 1740 (holotype K000231822!, isotype PRE0255283-0!).

Polygala leptophylla var. armata (Chodat) Paiva (1998: 260). Basionym: Polygala armata Chodat (1912: 327).

Type:-NAMIBIA. Dinter 2076 (lectotype SAM SAM0074087-0! designated here). Syntypes: NAMIBIA, Schäfer 69 \& 394. (not located)

Note:-Paiva designated Dinter 4953 (K000231823!, PRE0566780-0!) as neotype. However, at least a duplicate of a syntype (an isosyntype) exists which supersedes it. It is selected here as lectotype.

Polygala leucocarpa Chodat (1893: 400) [=P. pallida $]$

Type:-NAMIBIA. Schinz 507 (holotype Z). 
Note:- The holotype has not been located at Z.

Polygala levynsiana Paiva (1998: 272)

Type:-SOUTH AFRICA. Western Cape: Ladismith, Waterkloof, Levyns 9063 (holotype BOL).

Polygala ligularis Ker Gawler (1822: t. 637) [=P. myrtifolia $]$

TYPE:-SOUTH AFRICA. Masson s.n. (holotype BM).

Polygala livingstoniana Chodat (1893: 339) [=P. albida $]$

Syntypes:-ANGOLA. Massumba Pogge $27(\mathrm{~B} \dagger)$; NAMIBIA, Amboland, Schinz $506(\mathrm{G} \dagger)$;

SOUTH AFRICA, Transvaal, Hoggefeld, Rehmann 6731 (G†).

Note:- See note under $\boldsymbol{P}$. albida.

Polygala longifolia Presl (1845: 445) non Poiret in Lamarck \& Poiret (1804: 501), nom. illeg. $[=\boldsymbol{P}$. virgata]

Type:-SOUTH AFRICA. Drège 7211 (BM, CGE, E, HBG508728!, K, PR?, NY00444481!, OXF).

Polygala ludwigiana Ecklon \& Zeyher (1835: 23)

Type:-SOUTH AFRICA. Western Cape: Clanwilliam. Brakfontein, Ecklon \& Zeyher 175

(lectotype K [designated by Paiva 1998: 267 as 'holo-'], isolectotypes S08-16509!, SAM0029301$0 !)$.

Polygala lysimachiifolia Chodat (1893: 377)

Type:-SOUTH AFRICA. KwaZulu-Natal: Natal, Inanda, Wood 1496 (BM, G, NH).

Note:- In the protologue Chodat cites 'Ex Hb. Nat. Bot. Garden' which means 'from the Herbarium of Natal Botanic Garden', where Medley Wood was based. The holotype could be a specimen at $\mathrm{G}$, as Chodat was based at Geneve. We were unable to locate it in that herbarium. It was possibly destroyed during the 1898 fire that consumed Chodat's herbarium (Anon. 1899).

Polygala macowaniana Paiva (1998: 185)

Type:-SOUTH AFRICA. Eastern Cape: Griqualand East, Malowe Mt., Tyson 2082 (holotype K000231818!, isotypes E, PRE!, SAM0029323-2!, SAM0029323-1!). 
Polygala macra Candolle (1824: 323) [=P. umbellata $]$

Type:-SOUTH AFRICA. Burchell s.n. (holotype G-CD).

Polygala macrantha Turczaniow (1855: 345) [=P.fruticosa $]$

Type:-SOUTH AFRICA. Drège s.n. (lectotype KW KW001000993! designated here).

Note:-The specimen KW001000993 is listed as lectotype in JSTOR Global Plants (2013).

However, we were unable to locate where in the literature this designation was done. Possible

duplicates of the lectotype are at FR, KW001000945! and W).

Polygala macrophylla D.Dietrich (1847: 905)

Type:-Not designated.

Note:- It was published as a replacement name for P. longifolia A. Dietrich non Poiret in Lamarck \& Poiret (1804: 501), a nom. illeg. If the type exists, it could be at HBG where A. Dietrich's types are held.

Polygala marensis Burtt Davy (1926: 48)

Type:-SOUTH AFRICA. Limpopo: Transvaal, Zoutpansberg, Mara, Rogers 22616 (holotype K000231817!, isotype BM000564946!).

Polygala meridionalis Levyns (1955: 37)

Type:-SOUTH AFRICA. Western Cape: Bredasdorp Div., near Elim, Schlechter 9649 (holotype $\mathrm{B} \dagger$, lectotype BOL [designated by Levyns 1955: 37], isolectotypes BM000564942!, BR0000006273774!, COI00005895!, E00193038!, HBG508664!, K000231816!, S-G-4989!, WAG0002699!, W, Z-000019420!).

Polygala meyeriana Presl (1845: 444) [=P.fruticosa $]$

Type:-SOUTH AFRICA. Drege s.n. (holotype not located).

Note:-Presl types are mostly at PR (Prague).

Polygala microlopha Burchell ex Candolle (1824: 322)

Type:-SOUTH AFRICA. Eastern Cape: Albany Div., East of Zwatwater Poort, Burchell 3416 (holotype K000231815!). 
Polygala microlopha var. gracilis Levyns (1955: 27) [=P. levynsiana $]$

Type:--Same as P. levynsiana.

Polygala microphylla Thunberg (1800: 121) non Linnaeus (1763: 989) nom. illeg. [=P. thunbergii] Type:-SOUTH AFRICA. Thunberg s.n. (holotype UPS Nr 16229!).

Polygala mossii Exell (1928: 299)

Type:-NAMIBIA. Moss 11568 (holotype BM000564943!, isotype G00008727!).

Polygala myrtifolia Linnaeus (1753: 703)

Type:- Coll. details unknown (lectotype S-LINN, IDC [microfiche] no. 292. image!) [designated by Figueiredo et al. 2013]).

Polygala myrtifolia var. amoena (Thunb.) Harvey in Harvey \& Sonder (1860: 83) [=P. myrtifolia $]$. Basionym: Polygala amoena Thunberg (1800: 120)

Type:-Same as P. amoena.

Polygala myrtifolia var. cluytioides (Burch. ex DC.) Harvey in Harvey \& Sonder (1860: 83) [=P.myrtifolia]. Basionym: Polygala cluytioides Burchell ex Candolle (1824: 322)

Type:- Same as P. cluytioides

Polygala myrtifolia var. coriacea Chodat (1893: 422) non Lehmann (1830) nom. illeg. [=P. myrtifolia]

Type:-SOUTH AFRICA. Krebs s.n. (holotype B†).

Polygala myrtifolia var. grandiflora (Lodd.) Chodat (1893: 422) [=P. myrtifolia]. Basionym:

Polygala grandiflora Loddiges (1827: t.1227)

Type:-Same as $P$. grandiflora.

Polygala myrtifolia var. lancifolia Chodat (1893: 423) [=P. myrtifolia $]$

Syntypes:-SOUTH AFRICA. Drège 7186 (BM, E, HBG508729!, K, W), Ecklon \& Zeyher 143

$(\mathrm{M}, \mathrm{W})$. 
Polygala myrtifolia var. ligularis (Ker Gawl.) Harvey in Harvey \& Sonder (1860: 83) [=P.

myrtifolia]. Basionym: Polygala ligularis Ker Gawler (1822: t. 637)

Type:- Same as P. ligularis.

Polygala myrtifolia var.natalensis Sonder (1850: 14) [=P. myrtifolia $]$

Type:-SOUTH AFRICA. Gueinzius 288 (holotype S-G-4988!).

Polygala myrtifolia var. pinifolia (Lam. ex Poir.) Paiva (1998: 280). Basionym: Polygala pinifolia Lamarck ex Poiret in Lamarck \& Poiret (1804: 492).

Type:-SOUTH AFRICA. S.c. s.n. (holotype P-LA).

Polygala myrtifolia var. obtusifolia Chodat (1893: 423) [=P.myrtifolia]

Type:-SOUTH AFRICA. Boivin s.n. (lectotype E [designated by Paiva 1998: 279], isolectotype $\mathrm{W})$.

Polygala myrtifolia var. salicifolia (Chodat) Durand \& Schinz (1898: 239) [=P. myrtifolia $]$

Basionym: Polygala myrtifolia forma salicifolia Chodat (1893: 422)

Type:- Same as P. myrtifolia forma salicifolia.

Polygala myrtifolia var. vera Candolle (1824: 322) $[=\boldsymbol{P}$. myrtifolia $]$

Type:-Illustration in Ker Gawler (1822: P1. 669), lectotype designated here.

Polygala myrtifolia forma salicifolia Chodat (1893: 422) [=P. myrtifolia $]$

Type:-SOUTH AFRICA. Burchell 3535 (lectotype K [designated by Paiva 1998: 279 as 'holo-'], $\mathrm{M})$.

Polygala natalensis Chodat (1896: 237) [=P. serpentaria $]$

Type:-SOUTH AFRICA. KwaZulu-Natal: Natal , 1881, Saunders s.n. (holotype K000231789!).

Polygala nematocaulis Levyns (1955: 49)

Type:-SOUTH AFRICA. Western Cape: Caledon, Steenbras Siding, Levyns 8767 (holotype BOL136749!). 
Polygala nodiflora Chodat (1912: 325)

Type:-SOUTH AFRICA. Mpumalanga: Lydenburg, Wilms 42 (lectotype BM designated here).

Polygala nummularia Burchell ex Candolle (1824: 322) [=P.fruticosa]

Type:-SOUTH AFRICA. Burchell 3709 (lectotype K000231640! [designated by Paiva 1998:

281], isolectotype G-DC G00210282!, M)

Polygala ohlendorfiana Ecklon \& Zeyher (1835: 22)

Type:-SOUTH AFRICA. Eastern Cape: Orange Mts, Winterberg, Ecklon \& Zeyher s.n.

(lectotype M0104318![designated by Paiva 1998: 209]).

Note:-Ecklon \& Zeyher (1835) did not cite a collecting number in the protologue of Polygala

ohlendorfiana. The number 170 that appears on some specimens is the number of the species in the publication where it was described. Several duplicates exist, but because they are either unnumbered or numbered ' 170 ', they cannot be considered isolectotypes. These specimens are deposited at BREM, CGE, E, GOET009614!, HBG508666!, HBG508665!, JE00003235!, K000231811!, LD, MO-251877!, OXF, REG000943!, S08-16535!, S08-16532!, SAM00384060 ! and $\mathrm{W}$.

Polygala ophiura Chodat (1893: 376)

Syntypes:-SOUTH AFRICA. KwaZulu-Natal: Camperdown, Rehmann 7749 \& Oakfort, Umhloti, 8524 (not located), Inanda, Wood s.n.(not located)

Note:- None of the syntypes could be located. Paiva (1998) suggested that they might have been destroyed at B.

Polygala oppositifolia Linnaeus (1771: 259) nom. illeg. [=P. fruticosa $]$

Type:-SOUTH AFRICA. Tulbagh 138 (lectotype LINN LINN 882.15 [designated by Paiva 1998:

280 as 'holo']).

Note:- Jarvis (2007) considered that the type was not designated. However, Paiva (1998)

designated the specimen at LINN which was determined by Linnaeus. The name is a nom.illeg. as it is a replacement name for the earlier P. fruticosa P.J.Bergius.

Polygala oppositifolia var. borboniifolia (Burch. ex DC.) Harvey in Harvey \& Sonder (1860: 82)

[=P. fruticosa]. Basionym: Polygala borboniifolia Burchell ex Candolle (1824: 322,

as 'borboniaefolia') 
Type:- Same as P.borboniifolia.

Note:- P. oppositifolia is a nom. illeg.

Polygala oppositifolia var. cordata Harvey in Harvey \& Sonder (1860: 82) [=P. fruticosa $]$

Syntypes:-SOUTH AFRICA. Ecklon \& Zeyher 133a (M), 133b (K, W), 134 (HBG508658!, K, M, P, W), 135 (M0153002!, OXF, S08-16555!, S08-16553!, W).

Note:-P. oppositifolia is a nom. illeg.

Polygala oppositifolia var. cuspidata Harvey in Harvey \& Sonder (1860: 82) [=P. fruticosa]

Type:-SOUTH AFRICA. Ecklon \& Zeyher 132 (M, W).

Note:- P. oppositifolia is a nom. illeg.

Polygala oppositifolia var. lanceolata Harvey in Harvey \& Sonder (1860: 82) [=P. fruticosa $]$

Type:-SOUTH AFRICA. Drège s.n. (not determined)

Note:-Possible duplicates of this collection are: BM, E, HBG508690!, K, S08-16545! and W. P. oppositifolia is a nom. illeg.

Polygala oppositifolia var. latifolia Harvey in Harvey \& Sonder (1860: 82) [=P. fruticosa]

Type:-SOUTH AFRICA. Ecklon \& Zeyher 136 (M).

Note:-P. oppositifolia is a nom. illeg.

Polygala oppositifolia var. nummularia (Burch. ex DC.) Harvey in Harvey \& Sonder (1860: 82)

[=P. fruticosa]. Basionym: Polygala nummularia Burchell ex Candolle (1824: 322)

Type:- Same as P. nummularia.

Note:- P. oppositifolia is a nom. illeg.

Polygala oppositifolia var. rhombifolia (Eck1. \& Zey.) Harvey in Harvey \& Sonder (1860: 82) [=P. fruticosa]. Basionym: Polygala rhombifolia Ecklon \& Zeyher (1835: 18).

Type:-Same as $P$. rhombifolia.

Note:- P. oppositifolia is a nom. illeg.

Polygala oppositifolia var. trigonoides Ecklon \& Zeyher ex Harvey in Harvey \& Sonder (1860: 82) $[=P$. fruticosa $]$

Type:-SOUTH AFRICA. Drège s.n. (lectotype HBG HBG508697! designated here). 
Note:-The specimen designated here is part of the original material. Possible duplicates of this collection are: NY00444476, REG000945 and TUB001910. P. oppositifolia is a nom. illeg.

Polygala orthostigma Chodat (1914: 85) [=P. garcinii $]$

Type:-SOUTH AFRICA. Schlechter 11048 (lectotype K [designated by Paiva 1998: 271], isolectotypes BM, E, PRE!, W).

Polygala ovalis Meyer ex Drège (1843: 212) [=P. ohlendorfiana $]$

Type:-SOUTH AFRICA. Eastern Cape: Katberg, Drège s.n. (BM).

Polygala pallida Meyer ex Harvey in Harvey \& Sonder (1860: 93)

Type:-SOUTH AFRICA. Drège s.n. (holotype K000231806!, K000231807!, these are two small specimens on the same sheet).

Note:-Drège s.n. (S08-16561!), a fragment, is a possible duplicate of the type. Possible duplicates were also recorded at OXF and W by Paiva (1998).

Polygala pappeana Ecklon \& Zeyher (1835: 23)

Type:-SOUTH AFRICA. Western Cape: Tulbagh Valley, Ecklon \& Zeyher 176 (lectotype K000231804! [designated by Paiva 1998: 266 as 'holo-'], isolectotypes E00193039!, GOET009615!, M0104316!, MO-357174!, W)

Polygala parkeri Levyns (1955: 46)

Type:-SOUTH AFRICA. Western Cape: Stellenbosch, Somerset West, Parker 4282 (holotype BOL136748!, isotypes K, NBG0004193-0!).

Polygala parva Chodat (1912: 330) [=P. seminuda $]$

Type:-SOUTH AFRICA. Cooper 1292 (holotype Z).

Note:-Cooper 1292 is cited in the protologue of P. parva as type but it may be an error for Cooper 1392. Although neither of these collections is available at JSTOR Global Plants (2013) and could not be located at Z, Paiva (1998: 328) examined duplicates of Cooper 1392 at BM, K and W.

Polygala peduncularis Burchell ex Candolle (1824: 323)

Type:-SOUTH AFRICA. Western Cape: Knysna, Cloete's kraal, Burchell 5163 (lectotype K000231803! [designated by Paiva 1998: 274 as 'holo-'], PRE PRE0256347-0). 
Polygala pilifera Hoffmannsegg (1841: 82) [=P. bracteolata $]$

Type:-Unknown.

Note:- Name based on a cultivated plant (Paiva 1998). If type specimens exist, they could be located at B.

Polygala pinifolia Lamarck ex Poiret in Lamarck \& Poiret (1804: 492) [=P. myrtifolia var.

pinifolia]

Type:--Same as $\boldsymbol{P}$. myrtifolia var. pinifolia.

Polygala pottebergensis Levyns (1955: 30)

Type:-SOUTH AFRICA. Western Cape: Swellendam, Hamerkop, Levyns 8396 (holotype BOL).

Polygala praticola Chodat (1912: 332)

Type:-SOUTH AFRICA. KwaZulu-Natal: Natal, Rudatis 705 (holotype G, isotypes BM, COI, E, PRE0263068-0!, WAG0002705!)

Note:-The type is cited in the protologue as Rudatis 703, but it is Rudatis 705 . The holotype at G is not in JSTOR Global Plants (2013), but is extant in the herbarium.

Polygala producta Brown (1895: 142) emend. Burtt Davy (1924: 225)

Type:-SOUTH AFRICA. Mpumalanga: Barberton, Galpin 844 p.p. (lectotype K000231801! [designated by Burtt Davy 1924: 225]).

Note:-Several collections were cited by Brown (Galpin 844, Rehmann 4565, Bolus 3142, Burke 374, Nelson 281, Tyson 2741, Herb. Norm. Aust. Afr. 883, Gerrard 46, 1781, Wood 1171 and Sutherland s.n.), but Burtt Davy considered that only Galpin 844 and Rehmann 4565 belongs to the species. However, Galpin 844 is a mixed collection (Paiva 1998) that includes material of $P$. producta and $P$. stenopetala. The duplicate at $\mathrm{Z}$, the type of $P$. asperifolia, was determined by Paiva (1998) as P. stenopetala. Duplicates of Galpin 844 are recorded at SAM SAM0029327-0, BOL BOL136753 and NH NH0008728-0. These duplicates could not be examined to confirm their determinations.

Polygala pseudogarcinii Chodat (1912: 332)

Type:-SOUTH AFRICA. Western Cape: Riversdale, Rust 623 (not located).

Note:-The type could not be located and may have been destroyed. 
Polygala pubiflora Burchell ex Candolle (1824: 322)

Type:-SOUTH AFRICA. Western Cape: Mossel Bay, Burchell 6205 (lectotype K000231799!

[designated by Paiva 1998: 270 as 'holo-'], isolectotypes G-DC G00210298!, PRE0261556-0!).

Polygala pungens Burchell (1822: 304)

Type:-SOUTH AFRICA. Northern Cape: Buffelsbout, Burchell 1598 (holotype K000231798!).

Polygala pygmaea Gürke in Engler (1895: 234) [=P. welwitschii subsp. pygmaea]

Type:-Same as $\boldsymbol{P}$. welwitschii subsp. pygmaea

Note:-See under $\boldsymbol{P}$. welwitschii subsp. pygmaea

Polygala recognita Chodat (1893: 428)

Type:-SOUTH AFRICA. Western Cape: Cape Town, Bolus 2080 (lectotype BOL [designated by

Paiva 1998: 271], isolectotype B †). Syntypes: Schinz 81 (Z); Mundt s.n. (B); Elliot 128 (P).

Polygala reflexa Schinz (1908: 634) [=P. kalaxariensis]

Type:-NAMIBIA. Hereroland, Dinter 572 (lectotype Z Z-000019414! designated here, isolectotypes Z-000019426!, Z-000019425!).

Polygala refracta Candolle (1824: 323$)$

Type:-SOUTH AFRICA. Eastern Cape: Lange Kloof, near Joubertina, Burchell 4891 (lectotype K000231795! [designated by Paiva 1998: 267 as 'holo-'], isolectotypes G-DC G00210262!, PRE0259712-0!).

Polygala refracta var. steudeliana (Ecklon \& Zeyher) Harvey in Harvey \& Sonder (1860: 89) [=P triquetra]. Basionym: Polygala steudeliana Ecklon \& Zeyher (1835: 23)

Type:- Same as P. steudeliana.

Polygala rehmannii Chodat (1893: 362)

Type:-SOUTH AFRICA. Gauteng: Transvaal, Pretoria, Kuduspoort, Rehmann 4635 (holotype Z000025266!). 
Polygala rehmannii var. parviflora Chodat (1893: 362) [=P. producta $]$

Type:- SOUTH AFRICA. Gauteng: Pretoria, Wonderboompoort, Rehmann 4565 (holotype Z†, lectotype BM000566294! [designated by Paiva 1998: 221], isolectotype K000231802!).

Note:-The holotype has not been located at Z. It was considered destroyed Paiva (1998), who designated the specimen held at BM as lectotype.

Polygala retiefiana Paiva \& Figueiredo in Paiva et al. (2013:31)

Type:-SOUTH AFRICA. Free State: Harrismith District, Berg Manyenyeza op die plaas [on the farm] Rensburgskop, 2829AD, 1800 m elev., 17 January1977, Jacobsz 347 (holotype PRE!).

Polygala rhinostigma Chodat (1912: 328)

Type:-SOUTH AFRICA. KwaZulu-Natal: Natal, Umkomanzi, Schlechter 6700 (holotype Z, isotype GRA0001790-0!).

Polygala rhinostigma var. latialata Chodat (1912: 329) [?=P. rhinostigma $]$

Type:-SOUTH AFRICA. Mpumalanga: Transvaal, Drakensberg, Medley-Wood 3482 (holotype Z).

Polygala rhombifolia Ecklon \& Zeyher (1835: 18) [=P.fruticosa $]$

TYPE:-SOUTH AFRICA. Ecklon \& Zeyher 139 (lectotype W [designated by Paiva 1998: 281], isolectotype M0152999!).

Polygala rigens Burchell (1822: 465)

Type:-SOUTH AFRICA. Eastern Cape: Griqualand West, Burchell 1821 (holotype K000231794!, isotype G-DC G00210488!).

Polygala rodrigueana Paiva (1998: 247)

Type:-SOUTH AFRICA. KwaZulu-Natal: Natal , Wood s.n. (holotype Z-000019407!).

Polygala scabra Linnaeus (1753: 703) [=P.affinis $]$

Type:- Not designated, to be designated by Figueiredo et al. (in press).

Polygala schinziana Chodat (1893: 364)

Type:-NAMIBIA. Amboland, Schinz 502 (holotype Z-000083744!). 
Note:-The type is cited in the protologue (Chodat 1898) as Rehmann 502 (p. 364) and as Schinz 502 (page 365). The latter appears to be the correct citation.

Polygala schlechteri Schinz (1910: 237) [=P. spicata $]$

Type:-SOUTH AFRICA. Transvaal, Schlechter 4117 (lectotype BM000564933! [designated by Paiva 1998: 157], isolectotypes FR, HBG508708!, K000231785!, MO-251876!, PRE0255287-0!, W, Z-000019430!).

Polygala sekhukhuniensis Retief, S.J.Siebert \& A.E.Van Wyk in Siebert et al. (2010: 346)

Type:-SOUTH AFRICA. Mpumalanga: Pilgrim's Rest, Thornecliffe Chrome Mine, turn off from Lydenburg-Sekhukhune road, Van Wyk 13031 (holotype PRU, isotype PRE!).

Polygala seminuda Harvey in Harvey \& Sonder (1860: 86)

Type:-SOUTH AFRICA. Western Cape: Bushmanland, at Kamos Komsep and Springbokkeel, Zeyher 58 (lectotype K[designated by Paiva 1998: 260 as 'holo-'], isolectotypes BM000843955!, PRE0259825-0!, SAM0029280-0!). Syntype: Pappe 30 (TCD0002088!).

Polygala senensis Klotzsch in Peters (1862: 113)

Type:-MOZAMBIQUE. Sena, Peters s.n. (holotype B $†$ ).

Polygala serpentaria Ecklon \& Zeyher (1835: 24)

Type:-SOUTH AFRICA. Eastern Cape: nr Chumiberg and Beaufort, Kat River, Ecklon \& Zeyher 181 (lectotype M0152917 [designated by Paiva 1998: 246], isolectotypes MO-357111!, OXF, PRE0255286-0!, SAM0014196-1!, SAM0014196-2).

Polygala simplex Burchell ex Candolle (1824: 322) [=P. virgata var. decora]

Type:-SOUTH AFRICA. Burchell 1933 (holotype K000231775!).

Polygala spartioides (Burch. ex DC.) Ecklon \& Zeyher (1835: 20) [=P. virgata]. Basionym:

Polygala genistoides var. spartioides Burchell ex Candolle (1824: 323)

Type:- Same as $P$. genistoides var. spartioides.

Polygala speciosa Sims (1816: tab.1780) [=P. virgata var. speciosa]

Type:- Same as $\boldsymbol{P}$. virgata var. speciosa. 
Polygala sphenoptera Fresenius (1837: 274)

Type:-ETHIOPIA. between Halei and Tembien, Rüppell s.n. (holotype FR0030082!).

Polygala spicata Chodat (1893: 221)

Type:-ANGOLA. Welwitsch 1027 (holotype $\mathrm{B} \dagger$, lectotype LISU [designated by Paiva 1998: 157], isolectotypes BM000564444!, COI00005527!, K000231702!, P00375294!, PRE0265666-0!).

Polygala stenopetala Klotzsch in Peters (1861: 114) 'stenophylla'

Type:-MOZAMBIQUE. Inhambane, Peters s.n. (holotype B $\uparrow$, lectotype P [designated by Paiva 1998: 220]).

Polygala steudeliana Ecklon \& Zeyher (1835: 23) [=P. triquetra]

Type:-SOUTH AFRICA. Ecklon 648 (lectotype M0153116! [designated by Paiva 1998: 270], isolectotype TCD).

Note:-Two specimens at S, S08-16594 and S08-16595, are possibly duplicates of the lectotype.

The collection is often cited as number 180 (the number in the species list).

Polygala striata Thunberg (1800: 121)

Type:-SOUTH AFRICA. Thunberg s.n. (holotype UPS Nr 16250!).

Polygala subcarnosa Schinz (1931: 145) [=P. mossii $]$

Type:-NAMIBIA. Dinter 6372 (holotype Z-000019431!, isotypes BM, BOL136752!, HBG508671!, K000231814!).

Polygala teretifolia Linnaeus f. (1782: 316)

Type:-SOUTH AFRICA. Thunberg s.n. (holotype UPS Nr 16251!, isotypes LD1237884!, S0816661 ).

Polygala tetragona Burchell ex Candolle (1824: 322) [=P.fruticosa]

Type:-SOUTH AFRICA. Burchell 4639 (lectotype K K000231641! designated here, isolectotype G-DC G00210315).

Polygala thunbergii Candolle (1824: 333)

Type:-SOUTH AFRICA. Thunberg s.n. (holotype UPS Nr 16229!). 
Note:-A new name for P. microphylla Thunb., which was a nom. illeg.

Polygala tomentosa Thunberg (1800: 120) [=P. fruticosa $]$

Type:-SOUTH AFRICA. Thunberg s.n. (holotype UPS Nr 16256!, isotype S-G-4991!).

Polygala transvaalensis Chodat (1893: 374)

Type:-SOUTH AFRICA. Gauteng: Pretoria, Aapies Poort, Rehmann 4198 (lectotype

K000231781![designated by Paiva 1998: 245], isolectotype Z). Syntype: Rehmann 6348 (Z).

Note:-The two duplicates could not be located at Z.

Polygala triquetra Presl (1845: 445)

Type:-SOUTH AFRICA. Drège 7193 (lectotype K000231780! [designated by Paiva 1998: 270 as 'holo-'], isolectotypes BM, G, PRE0261491-0!, W).

Note:-We could not determine if there is a holotype at PR, which would take precedence over the lectotype.

Polygala ukambica Chodat (1893: 329) [=P. sphenoptera $]$

Type:-SOUTH AFRICA. KwaZulu-Natal, Natal, Weenen, Wood 4433 (lectotype NH

NH0005028-0! designated here, isolectotype G). Syntype: Kenya, Hildebrandt 2785 (G).

Polygala ukambica var. transvaalensis Chodat (1893: 329) [=P. sphenoptera]

Type:-SOUTH AFRICA. Limpopo: Transvaal, Boshveld, Klippan, Rehmann 5216 (holotype Z000083749!, isotypes BM, K000231787!, Z-000083748!).

Polygala ukambica var. transvaalensis forma angustifolia Chodat $(1893: 330)[=\boldsymbol{P}$.

sphenoptera]

Type:-SOUTH AFRICA. Limpopo: Transvaal, Houtbosh, Rehmann 6346 (holotype Z000083747!, isolectotypes BM, K000231786!).

Polygala ukambica var. transvaalensis forma condensata Chodat (1893: 330) [=P. sphenoptera]

Type:-SOUTH AFRICA. Transvaal, Boshveld, Elandsriver, Rehmann 4943 [ '1943 ']

(lectotype K K000231788! designated here, isolectotype BM). 
Polygala umbellata Linnaeus (1771: 259)

Type:-SOUTH AFRICA. Tulbagh 163 (lectotype LINN 882.13! [designated by Levyns 1955:30]).

Polygala uncinata Meyer ex Meisner (1842: 468)

Type:-SOUTH AFRICA. Eastern Cape: Komgha, banks of Kei River, Drège s.n. (lectotype [firststep] K [designated by Paiva 1998: 218], lectotype [second step] K000231778! designated here). Note:-Exell (1960: 303) stated that the type of $P$. hamata was the same as that of $P$. uncinata, and that it originated from 'S. Africa (Port Natal)'. Later Paiva (1998: 218) designated a collection, Drège s.n. (K) from 'Natal, Port Natal', as lectotype of P. hamata. However, all the Drège specimens of both P. uncinata and P. hamata imaged at JSTOR Global Plants (2013) originate from the Kei River area, and not from Port Natal. In the protologue of P. hamata, Burtt Davy (1926: 135) cited the Kei River locality, and he did not record the occurrence of the species in Natal (now KwaZulu-Natal). It is possible that Exell was referring to the collection Krauss 216 from Port Natal, which is cited in the protologue of P. uncinata. Two specimens of Drège s.n. exist at $\mathrm{K}$ (K000231778 and K000231776). The one registered as K000231778 is here designated as lectotype (second-step lectotype).

Polygala virgata Thunberg (1800: 120)

Type:-SOUTH AFRICA. Cape, Thunberg s.n. (holotype UPS Nr 16262!).

Polygala virgata var. cernua (Thunb.) Chodat (1893: 404) [=P. virgata $]$ Type:-Same as P. cernua.

Polygala virgata var. decora (Sond.) Harvey in Harvey \& Sonder (1860: 85). Basionym: Polygala decora Sonder (1850: 14)

Type:-SOUTH AFRICA. KwaZulu-Natal: Port Natal, Gueinzius 23 (holotype S08-1082!, isotypesS S08-1081!, S-G-4985!).

Polygala virgata var. genistoides (Poir.) Harvey in Harvey \& Sonder (1860: 85) [=P. virgata $]$. Basionym: Polygala genistoides Poiret in Lamarck (1804: 492)

Type:--Same as $P$. genistoides. 
Polygala virgata var. intermedia Harvey in Harvey \& Sonder (1860: 85) [=P. virgata]

Type:-SOUTH AFRICA. Drège 7211 (lectotype K [designated by Paiva 1998: 249 as 'holo-'], isolectotypes BM, CGE, E, HBG508728!, OXF, NY00444481!, W)

Polygala virgata var. speciosa (Sims) Harvey in Harvey \& Sonder (1860: 85) pro parte (see Paiva 1998: 250). Basionym: Polygala speciosa Sims (1816: tab.1780)

Type:-SOUTH AFRICA. Western Cape: near Cape Town, Masson s.n. (lectotype BM000843962! [designated by Paiva 1998: 250]).

Polygala welwitschii Chodat (1893: 341)

Type:-ANGOLA. Welwitsch 1015 (holotype B†), Welwitsch 1008 (neotype BM000564928! [designated by Paiva 2007: 35, wrongly given as '1007'], isoneotypes LD, LISU, M0153125!). Notes:- In the protologue of Polygala welwitschii, Chodat (1893:341) cited only one collection, Welwitsch 1015, which he considered as including material of var. linearifolia and var. ovatifolia, thus the types of these two varieties are both Welwitsch 1015 p.p. Chodat (1893) also cited the same Welwitsch 1015 as type of $P$. stanleyana Chodat (1893: 340), but that might have been a mistake for Welwitsch 1017, which at BM bears Chodat's determination as P. stanleyana. Exell (1957) considered the holotype of P. stanleyana to be Welwitsch 1015 (B), which was destroyed during WWII, and he designated Welwitsch 1017 (BM) as 'lectotype'. As noted by Paiva (2007) this is rather a neotype because it is not cited in the protologue.

The duplicates of Welwitsch 1015 at B are considered the holotypes of these names (Exell 1957) but none is extant. According to Paiva (1998) four duplicates exist at BM and LISU but only one duplicate is imaged at JSTOR Plant Science (2013).

Welwitsch 1015 (BM000564929!) has a handwritten determination by Chodat as $P$. welwitschii var. ovatifolia and this specimen is here designated as lectotype. Welwitsch 1008 (BM000564928!) was determined by Chodat as $P$. welwitschii var. linearifolia. Since var. linearifolia is considered a nom. illeg. and the correct name is var. welwitschii, Welwitsch 1008 is the type of the species name. Paiva (2007) designated Welwitsch 1008 (BM) as lectotype, but wrongly gave the number as ' 1007 '. This is also rather a neotype since Chodat did not refer to that number in the protologue. The same specimen [Welwitsch $1008(\mathrm{BM})]$ was also designated by Paiva (1998: 189) as the lectotype of $P$. arenaria var. andongensis Oliver (1868: 128). 
Polygala welwitschii var. linearifolia Chodat (1893: 341) nom. illeg. [=P. welwitschii]

Type:-ANGOLA. Welwitsch 1015 p.p. (holotype B†, BM?, LISU?).

NOTE:-See under $\boldsymbol{P}$. welwitschii.

Polygala welwitschii var. ovatifolia Chodat (1893: 341) [=P. welwitschii $]$

Type:-ANGOLA. Welwitsch 1015 p.p. (holotype B†, lectotype BM000564929! designated

here, isolectotype LISU?).

Note:-See under $\boldsymbol{P}$. welwitschii.

Polygala welwitschii subsp. pygmaea (Gürke) Paiva (1998: 189). Basionym: Polygala pygmaea Gürke in Engler (1895: 234)

Type:-TANZANIA. Bukoba District, Bukoba, Stuhlmann 3763 (holotype B $\dagger$, lectotype [second-step] K K000231716! designated here, isolectotypes BM000843854!, K000231717!).

Note:-Although a lectotype was designated by Paiva (1998: 189) as being deposited at K, two duplicates exist in that herbarium and none has a label indicating it as a lectotype. For this reason a lectotype is designated here, from one of the two duplicates (second-step lectotype).

Polygala westii Exell (1957: 13)

Type:-ZIMBABWE. Matobo, Westacre Creek, West 2702 (holotype BM000564930!, isotype SRGH0106485-0!).

Polygala wilmsii Chodat (1912: 329)

Type:-SOUTH AFRICA. Mpumalanga: Transvaal, Lydenburg, Wilms 45 (holotype Z, isotypes BM000843858!, E, HBG508727!, K000231771!, M0104305!, P).

Polygala wilmsii var. subcapitata Chodat (1912: 329) [?=P. wilmsii]

Type:-SOUTH AFRICA. Transvaal, Schlechter 4656 (not located).

Note:-The collection Schlechter 4656 has not been located.

Polygala wittebergensis Compton (1931: 298)

Type:-SOUTH AFRICA. Western Cape: Laingsburg, Witteberg, Compton 3287 (holotype BOL). 
Polygala woodii Chodat (1896: 237) 'woodei'

Type:-SOUTH AFRICA. KwaZulu-Natal: Natal, Inanda, Wood 197 (lectotype K000231770!

[designated by Paiva 1998: 218], isolectotype BM000566296!).

Polygala xerophytica Chodat (1912: 329) [=P. ephedroides $]$

Syntypes:-NAMIBIA. Range $469(\mathrm{~B} \dagger)$, Trotha $142(\mathrm{~B} \dagger)$, Schultze $446(\mathrm{~B} \dagger)$, Range $674(\mathrm{~B} \dagger)$.

Polygala zahlbruckneri Hayek (1900: 45) [=P. garcinii $]$

Type:-SOUTH AFRICA. Penther 1482 (holotype W).

\section{ACKNOWLEDGEMENTS}

We thank Dr Laurent Gautier (G) and Dr Reto Nyffeler $(Z)$ for information on the collections of Polygala held at G and Z.

\section{REFERENCES}

Anonymous (1899). News. The American Naturalist 33 (389): 455-459.

Bergius, P.J. (1767). Descriptiones Plantarum ex Capite Bonae Spei. Laur. Salvii, Stockholm. 360 pp.

Brown, N.E. (1895). 218. Polygala producta. Bulletin of Miscellaneous Information Kew. 1895: 142.

Burchell, W. (1822). Travels in the interior of Southern Africa 1. Longman, Hurst, Rees, Orme, Brown and Green, London, 614 pp.

Burtt Davy, J. (1924). New or Noteworthy South African Plants.: VI. Bulletin of Miscellaneous Information Kew 1924: 223-235.

Burtt Davy, J. (1926). A Manual of the Flowering Plants and Ferns of the Transvaal with Swaziland, South Africa. Part 1. Longmans, Green and Co., London. 269 pp.

Candolle, A.P. de (1824) Prodromus systematis naturalis regni vegetabilis, 1. Treuttel \& Würtz Paris, $747 \mathrm{pp}$.

Chodat, R.H. (1889). Contributions à la flore de Paraguay. Mémoires de la Société de Physique et d'Histoire Naturelle de Genève 30: 103-115.

Chodat, R.H. (1893). Monographia Polygalacearum. Mémoires de la Société de Physique et d'Histoire Naturelle de Genève 31(2): 1-500.

Chodat, R.H. (1896). Polygalaceae novae vel parum cognitae. Bulletin de l'Herbier Boissier 4: 233-237. 
Chodat, R.H. (1912). Polygalaceae africanae IV. Botanische Jahrbucher für Systematik, Pflanzengeschichte und Pflanzengeographie 48: 309-336.

Chodat, R.H. (1914). Polygalaceae novae. Botanische Jahrbücher für Systematik, Pflanzengeschichte und Pflanzengeographie 52, Beibl. 115: 70-85.

Compton, R.H. (1931). The Flora of the Whitehill District. Transactions of the Royal Society of South Africa 19: 269-329.

Dietrich, A. (1834). Die Polygaleae. Allgemeine Gartenzeitung 2: 113-123.

Dietrich, D. (1847). Synopsis Plantarum seu enumeratio systematica plantarum, volume 4. Weimar, B. F. Voigt, [731-] 1692 pp.

Drège, J.F. (1843). Zwei Pflanzengeographische Documente. Unknown publisher Leipzig, 16 pp.

Durand, T.A. \& Schinz, H. (1898). Conspectus Florce Africee, volume 1.Jardin Botanique de l'État, Brussels, $268 \mathrm{pp}$.

Ecklon, C.F. \& Zeyher, C. (1835). Enumeratio Plantarum Africae Australis extratropicae qua collectae, determinatae et expositae, volume 1. Perthes \& Besser, Hamburg, 145 pp.

Engler, A. (1894). Plantae Gürichianae. Ein Beitrag zur Kenntnis der Flora von Deutschsüdwestafrika. Botanische Jahrbücher fur Systematik, Pflanzengeschichte und Pflanzengeographie 19: 128-152.

Engler, A. (1895). Die Pflanzenwelt Ost-Afrikas und der Nachbargebiete. Teil C. Geographische Verlagshandlung Dietrich Reimer, Berlin, 433 pp.

Exell, A. W. (1928). A new species of Polygala from South-West Africa. Journal of Botany 66: 299.

Exell, A.W. (1957). Polygalae novae. Boletim da Sociedade Broteriana, Ser. 2, 31: 5-14.

Exell, A.W. (1960). Polygalaceae. IN: Exell, A.W. \& Wild, H. (eds) Flora Zambesiaca volume 1, Crown Agents for Overseas Governments and Administration, London, p. 303-336.

Ficalho, C. \& Hiern, W.P. (1881). On Central-African Plants collected by Major Serpa Pinto. Transactions of the Linnean Society of London, Botany 2: 11-36.

Figueiredo, E., Paiva. J. \& Smith, G.F. (2013). Typification of two Linnean names in Polygala (Polygalaceae): P. bracteolata and P. myrtifolia. Taxon 62: 807-808.

Figueiredo, E., Paiva. J. \& Smith, G.F. (in press). The reinstatement of Polygala affinis DC. and the identity of Polygala scabra L. (Polygalaceae). Bothalia.

Fresenius, J.P.G.W. (1837). Beitäge zur Flora von Abissinien. Abhandlungen aus dem Gebiete der Beschreibenden Naturforschenden Gesellschaft in Frankfurt am Main 2: 265-286.

Gandoger, M. (1913). L'Herbier Africain de Sonder. Bulletin de la Société botanique de France 60: 454-462. 
Geneva Herbaria Catalogue (2013). Catalogue des herbiers de Genève (CHG).

Conservatoire \& Jardin botaniques de la Ville de Genève. http://www.villege.ch/musinfo/bd/cjb/chg/index.php?lang=en [accessed in March 2013]

Harvey, W.H. \& Sonder, O.W. (1860). Flora Capensis, volume 1. Hodges, Smith \& Co., Dublin, 546 pp.

Hasskarl, J. (1864). Polygalaceae, praesertim indicae. Annales Musei Botanici Lugduno-Batavi 1: 142-196.

Hayata, B. (1908), Polygala armata. Journal of the College of Science, Imperial University of Tokyo 25: Art. 19, 54.

Hayek, A. (1900). Polygalaceae. IN: Zahlbruckner, A. Plantae Pentherianeae. Annalen des K. K. Naturhistorischen Hofmuseums 15: 42-46.

Hoffmannsegg, J.C. (1841). Polygala pilifera. Preiss-Verzeichniss der Pflanzen im Gräfl. Hoffmannseggischen Garten zu Dresden, ed. X: 82.

JSTOR Global Plants (2013). http://plants.jstor.org/ [accessed in March 2013]

Jarvis, C. (2007). Order out of chaos. Linnean plant names and their types. The Linnean Society $\&$ The Natural History Museum, London, 1016 pp.

Ker Gawler, J.B. (1822). Polygala latifolia, Polygala ligularis, Polygala myrtifolia. The Botanical Register 8: T.637, T.645, T.669.

Kew Herbarium Catalogue (2013). http://apps.kew.org/herbcat/navigator.do [accessed in March 2013]

Lamarck, J.-B.P.A.M. \& Poiret, J.L.M. (1804). Encyclopédie méthodique: botanique. Volume 5. H.Agasse, Paris, 758 pp.

Lehmann, J. (1830). Delectus seminum, quae in horto Hamburgensium botanico e collectione anni 1830 mutuae commutationi offeruntur. Hamburg.

Levyns, M. (1955). The species of Polygala in the south-western Cape Province. The Journal of South African Botany 21: 9-49.

Linnaeus, C. (1753). Species Plantarum. Impensis Laurentii Salvii, Stockholm, 1200 pp.

Linnaeus, C. (1763). Species Plantarum, edition 2, volume 2. Laurentii Salvii, Stockholm, 1682 pp.

Linnaeus, C. (1771). Mantissa Plantarum 2 Altera. Laurentii Salvii, Stockholm, 587 pp.

Linnaeus, C. (1782). Supplementum Plantarum Systematis Vegetabilium. Orphanotrophei, Brunsviga, 468 pp.

Loddiges, C. (1820). Botanical Cabinet; Consisting of Coloured Delineations of Plants from all Countries, volume 5. Loddiges \& Sons, London. 
Loddiges, C. (1824). Botanical Cabinet; Consisting of Coloured Delineations of Plants from all

Countries, volume 10. J. \& A. Arch, Longman, Rees, Orne, Brown \& Green, \& Loddiges \& Sons, London.

Loddiges, C. (1827). Botanical Cabinet; Consisting of Coloured Delineations of Plants from all Countries, volume 13. J. \& A. Arch, Longman, Rees, Orne, Brown \& Green, \& Loddiges \& Sons, London.

MacOwan, P. (1890). New Cape Plants. Journal of the Linnean Society Botany, 25: 385-394.

Markötter, E.I. (1930). 'n Plantegeografiese skets en die flora van Witzieshoek, O.V.S.; Oliviershoekpas, Natal; en Koolhoek, O.V.S. Annale van die Universiteit van Stellenbosch 8, A, 1: 1-50.

McNeill, J., Barrie, F.R., Buck, W.R., Demoulin, V., Greuter, W., Hawksworth, D.L., Herendeen, P.S., Knapp, S., Marhold, K., Prado, J., Prud'homme van Reine, W.F., Smith, G.F., Wiersema, J.H. \& Turland, N.J. (eds.). (2012). International Code of Nomenclature for algae, fungi, and plants (Melbourne Code) adopted by the Eighteenth International Botanical Congress Melbourne, Australia, July 2011. Koeltz Scientific Books, Königstein. [Regnum Vegetabile 154], 208 pp.

Meisner, C.F. (1842). Contributions towards a Flora of South Africa. The London Journal of Botany 1: 459-476.

Nuttall, T. (1818). The Genera of North American Plants. Publ. by author, Philadelphia, 312 pp.

Oliver, D. (1868). Flora of Tropical Africa. Volume 1. L. Reeve, London, 479 pp.

Paiva, J. (1998). Polygalarum africanarum et madagascariensium prodromus atque gerontogaei generis Heterosamara Kuntze, a genere Polygala L. segregati et a nobis denuo recepti, synopsis monographica. Fontqueria 50: 1-346.

Paiva, J. (2007) Polygalaceae. IN: Beentje, H. J. \& Ghazanfar, S. A. (eds.) Flora of Tropical East Africa. Royal Botanic Gardens, Kew, 64 pp.

Paiva, J., Figueiredo, E. \& Smith, G.F. (2013) Polygala retiefiana (Polygalaceae), a new species described from South Africa. Phytotaxa 117(1): 30-34.

Peters, W.C.H. (1862). Naturwissenschaftiche Reise nach Mossambique volume 1. G. Reimer, Berlin, $304 \mathrm{pp}$.

Presl, C.B. (1845). Polygala. Abhandlungen der Königlichen Böhmischen Gesellschaft der Wissenschaften ser. 5, 3: 445.

Rendle, A.B., Baker, E.G., Moore, S. \& Gepp, A. (1911). A contribution to our knowledge of the flora of Gazaland. The Journal of the Linnean Society, Botany 40: 1-245. 
Schinz, H. (1888). Beiträge zur Kenntnis der Flora von Deutsch-Südwest-Afrika und der angrenzenden Gebiete I. Verhandlungen des Botanischen Vereins für die Provinz Brandenburg 29: 44-64.

Schinz, H. (1908). Polygalaceae. Bulletin de l'Herbier Boissier 8: 634.

Schinz, H. (1910). Beiträge zur Kenntnis der afrikanischen Flora (XXIII). Vierteljahrsschrift der Naturforschenden Gesellschaft in Zürich 55: 233-247.

Schinz, H. (1931). Polygala subcarnosa. Vierteljahrsschrift der Naturforschenden Gesellschaft in Zürich 76: 145.

Schlechter, R. (1898). Decades plantarum novarum austro-africanarum. Journal of Botany 36: 2328.

Sibthorp, J. \& Smith J.E. (1813). Florae Graecae prodromus. London, Richard Taylor, 422 pp.

Siebert, S.J., Retief, E., van Wyk, A.E. \& Struwig, M. (2010). A new species of Polygala (Polygalaceae) from ultramafic soils in Sekhukhuneland, South Africa, with notes on its ecology. South African Journal of Botany 76(2): 345-353.

Sims, J. (1816). Polygala speciosa. Botanical Magazine 43: Tab. 1780.

Smith, G.F. \& Figueiredo, E. (2013). Type specimens online: What is available, what is not, and how to proceed; Reflections based on an analysis of the images of type specimens of southern African Polygala (Polygalaceae) accessible on the worldwide web. Taxon 62: 801-806.

Sonder, W. (1850). Beiträge zur Flora von Sudafrica. Linnaea 23: 1-138.

The Linnean Collections (2013). http:// www.linnean-online.org/ [accessed in March 2013]

Thiers , B. (2013). Index Herbariorum. The New York Botanical Garden, New York. Available http://sciweb.nybg.org/science2/IndexHerbariorum.asp [accessed in March 2013]

Thunberg, C.P. (1800). Prodromus plantarum Capensium volume 2. Litteris Joh. Fr. Edman, Reg. Acad. Typogr., Uppsala, 192 pp.

Turczaninow, N.S. (1854). Animadversiones ad primam partem herbarii Turczaninowiani, nunc Universitatis Caesareae Charkowiensis. Bulletin de la Société Imperiale des Naturalistes de Moscou 27 (3): 272-372.

Uppsala Herbarium (UPS) Database (2013). http://130.238.83.220/botanik/findrecords.php?link=Find [accessed in March 2013]

Walter, T. (1788). Flora Caroliniana. J. Frazer, London, 263 pp.

Zürich Herbaria Database (2013). http://www.zuerich-herbarien.unizh.ch/ [accessed in March 2013] 